\title{
ON SOME WEAK CONDITIONS OF COMMUTATIVITY IN COMMON FIXED POINT THEOREMS
}

\author{
M. IMDAD \\ Aligarh Muslim University \\ Department of Mathematics \\ Aligarh 202001, India \\ M.S. KHAN \\ King Abdul Aziz University \\ Faculty of Science \\ Department of Mathematics \\ Jeddah 21413, Saudi Arabia \\ and

\section{S. SESSA}
Universita' di Napoli Facolta' di Architettura Istituto Matematico Via Monteoliveto 3 \\ 80134 Napoli, Italy \\ (Received September 2, 1986 and in revised form December 4, 1986)
}

ABSTRACT. We generalize common fixed point theorems of Fisher and Sessa in complete metric spaces, using some conditions of weak commutativity between a setvalued mapping and a single-valued mapping. Suitable examples prove that these conditions do not imply each of the others.

KEYS WORDS AND PHRASES. Common fixed point, Set-valued mapping, Weak commutativity. 1980 AMS SUBJECT CLASSIFICATION CODE. 54C60, 54 H25.

1. INTRODUCTION.

In this paper $(X, d)$ denotes a complete metric space and $B(X)$ stands for the set of all nonempty bounded subsets of $X$. The function $\delta$ of $B(X) \times B(X)$ into $[0$, +o) is defined as

$$
\delta(A, B)=\sup \{d(a, b): \quad a \in A, b \in B\}
$$

for all $A, B$ in $B(X)$. If $A=\{a\}$ is singleton, we write $\delta(A, B)=\delta(a, B)$ and if $B=\{b\}$, then we put $\delta(A, B)=d(a, b)$. It is easily seen that

$$
\begin{aligned}
& \delta(A, B)=\delta(B, A) \geqq 0, \\
& \delta(A, B) \leqq \delta(A, C)+\delta(C, B), \\
& \delta(A, A)=\text { diam } A, \\
& \delta(A, B)=0 \text { implies } A=B=\{a\}
\end{aligned}
$$


for all $A, B, C$ in $B(X)$. We recall some definitions and a basic lemma of Fisher [1]. Let $\left\{A_{n}: n=1,2, \ldots\right\}$ be a sequence of ncnempty subsets of $X$. We say that the sequence $\left\{A_{n}\right\}$ converges to a subset $A$ of $X$ if each point $a$ in $A$ is the limit of a convergent sequence $\left\{a_{n}\right\}$ with $a_{n}$ in $A_{n}$ for $n=1,2, \ldots$ and if for any $\varepsilon>0$, there exists an integer $N$ such that $A_{n} \subseteq A_{\varepsilon}$ for $n>N, A_{\varepsilon}$ being the union of all open spheres with centers in $A$ and radius $\varepsilon$. The following lemma holds.

LEMMA 1. If $\left\{A_{n}\right\}$ and $\left\{B_{n}\right\}$ are sequences of bounded subsets of (X,d) which converge to the bounded subsets $A$ and $B$ respectively, then the sequence $\left\{\delta\left(A_{n}, B_{n}\right)\right\}$ converges to $\delta(A, B)$.

A set-valued mapping $F$ of $X$ into $B(X)$ is continuous at the point $x$ in $X$ if whenever $\left\{x_{n}\right\}$ is a sequence of points of $X$ converging to $x$, the sequence $\left\{F x_{n}\right\}$ in $B(X)$ converges to $F x . F$ is said to be continuous in $X$ if it is continuous at each point $x$ in $X$. We say that $z$ is a fixed point of $F$ if $z$ is in $F z$.

Following the notations of our foregoing paper [2], we denote by $\psi$ the set of all real functions $\psi$ of $[0,+\infty)$ into $[0,+\infty)$ such that $\psi$ is nondecreasing, right continuous and $\psi(t)<t$ for all $t>0$.

2. SOME COMMENTS.

Let $I$ be a mapping of $X$ into itself such that

$$
F(X) \subseteq I(X)
$$

Let $x_{0}$ be an arbitrary point of $x$ and let $z_{0}$ be an arbitrary point chosen in $\mathrm{Fx}_{0^{\circ}}$. Since (2.1) holds, let $x_{1}$ be a point in $X$ such that $I x_{1}=z_{0}$. Having defined the point $x_{n}$ and chosen an arbitrary point $z_{n}$ in $F x_{n}$, then we can define inductively the sequence $\left\{x_{n}\right\}$ such that $I x_{n+1}=z_{n} \varepsilon F x_{n}$ for $n=0,1,2 \ldots$

Using this iterative process, Fisher [3] proved the following result.

THEOREM 1. Let $F$ be a mapping of $X$ into $B(X)$ and let $I$ be a continuous mapping of $X$ into itself satisfying the inequality

$$
\delta(F x, F y) \leqq c \cdot \max \{d(I x, I y), \delta(I x, F x), \delta(I y, F y), \delta(I x, F y), \delta(I y, F x)\}
$$

for all $x, y$ in $x$, where $0 \leqq c<1$. If FIx $=$ IFx for all $x$ in $x$ and if (2.1) holds, then $F$ and $I$ have a unique common fixed point $z$ and further $\mathrm{Fz}=\{\mathbf{z}\}$.

Fisher [1] also proved that

THEOREM 2. Theorem 1 holds if one assumes the continuity of $F$ in $X$ instead of the continuity of $I$.

The proof of both these theorems are based on the fact that the sequence $\left\{\delta\left(\mathrm{Fx}_{\mathrm{n}}, \mathrm{Fx}_{1}\right): \mathrm{n}=0,1,2, \ldots\right\}$ is bounded for any $\mathrm{x}_{\mathrm{o}}$ in $\mathrm{x}$. As in [2], from now on we assume that

$$
\sup \left\{\delta\left(\mathrm{Fx}_{\mathrm{n}}, \mathrm{Fx}_{1}\right): \mathrm{n}=0,1,2 \ldots\right\}<+\infty
$$

for some point $x_{0}$ in $X$. Further in [2], we defined two mappings $F$ and $I$ to be weakly commuting if $\operatorname{IFx} \varepsilon \mathrm{B}(\mathrm{X})$ and

$$
\delta(F I x, I F x) \leqq \max \{\delta(I x, F x) \text {, diam } I F x\}
$$

for all $x$ in $x$. Clearly two commuting mappings $F$ and $I$ are weakly commuting, but in general two weakly commuting mappings do not commute as it is shown in the Example 1 of [2]. 
Using this concept, the first part of Theorem 3.1 of [2], that generalizes Theorem 1 , runs as follows.

THEOREM 3. Let $F$ be a mapping of $X$ into $B(X)$ and let $I$ be a continuous mapping of $X$ into itself satisfying the inequality

$$
\delta(F x, F y) \leqq \psi(\max \{d(I x, I y), \quad \delta(I x, F x), \quad \delta(I y, F y), \quad \delta(I x, F y), \quad \delta(I y, F x)\})
$$

for all $\mathrm{x}, \mathrm{y}$ in $\mathrm{X}$, where $\psi \varepsilon \Psi$. If there exists a point $\mathrm{x}_{0}$ in $\mathrm{X}$ satisfying condition (2.3), if $F$ and $I$ weakly commute and if (2.1) holds, then $F$ and I have a unique common fixed point $z$ and further $F z=\{z\}$.

Note that if we assume $\psi(t)=c \cdot t$ for all $t \geqq 0$ and $0 \leqq e<1,(2.5)$ becomes (2.2). Following Itoh and Takahashi [4], we also consider two mappings $F$ and $I$ such that IFx $\subseteq F I x$ for all $x$ in $X$. In this case, we say that $F$ and $I$ quasi commute and it is evident that if $F$ and $I$ commute, they also quasi commute.

When we wrote the paper [2], we were unaware about the result of [1] and, under the same assumptions of Theorem 3, in the second part of Theorem 3.1 of [2], we assumed the continuity of $F$ instead of the continuity of $I$. In [2], we established the following inequality (see inequality (3.6) of [2]),

$$
\mathrm{d}\left(\mathrm{z}_{\mathrm{m}}, \mathrm{z}_{\mathrm{n}}\right) \leqq \delta\left(\mathrm{z}_{\mathrm{m}}, \mathrm{Fx_{n }}\right) \leqq \delta\left(F \mathrm{x}_{\mathrm{m}}, \mathrm{Fx} \mathrm{n}_{\mathrm{n}}\right)<\varepsilon
$$

for any $m, n>p, p$ being a suitable nonnegative integer. This inequality implies that $\left\{z_{n}\right\}$ is a Cauchy sequence, which converges to a point $z$ in $X$ since $X$ is complete. Unfortunately, the second part of the proof of Theorem 3.1 of [2] was not correctly established. Strictly speaking, the gap consists in the fact that (2.6) does not imply the following inequality of [2],

$$
\delta\left(z, F z_{n}\right) \leqq d\left(z, z_{n}\right)+\delta\left(z_{n}, F z_{n}\right) \leqq d\left(z, z_{n}\right)+\varepsilon
$$

for $n>p$, from which, as $n \rightarrow+\infty$, one should deduce that $F z=\{z\}$.

Here we point out that the second part of Theorem 3.1 of [2] can be substituted by the following result, which is a direct generalization of Theorem 2 .

THEOREM 4. Let $F$ be a continuous mapping of $X$ into $B(X)$ and let $I$ be a mapping of $X$ into itself satisfying the inequality (2.5) for all $x, y$ in $X$, where $\psi \varepsilon \Psi$. If there exists a point $x_{0}$ in $x$ satisfying condition (2.3), if $F$ and I quasi commute and if (2.1) holds, then $F$ and $I$ have a unique common fixed point $z$ and further $F z=\{z\}$.

PROOF. It is a minor variant of the proof of Theorem 1 of [1] and we omit it for brevity.

Note that if $F$ and $I$ commute and $\psi(t)=c \cdot t$ for all $t \geqq 0,0 \leqq c<1$, we deduce Theorem 2 from Theorem 4.

3. EXAMPLES.

The following example proves the greater generality of Theorem 4 over Theorem 2 .

EXAMPLE 1. Let $\mathrm{X}=[0,1]$ with the function $\delta$ induced by the Euclidean metric $d$. Define the mappings $F$ and $I$ as $F x=[0, x /(x+4)]$ for all $x$ in $x$ and $I x=x / 2$ if $0 \leqq x \leqq 2 / 3$ and $I x=1$ if $2 / 3<x \leqq 1$. Then we have that 


$$
F(X)=\left[0, \frac{1}{5}\right] \subseteq\left[0, \frac{1}{3}\right] \cup\{1\}=I(X)
$$

and

$$
\operatorname{IFx}=[0, x /(2 x+8)] \subseteq\left\{\begin{array}{l}
{[0, x /(x+8)]=F I x \text { if } 0 \leqq x \leqq 2 / 3} \\
{[0,1 / 5]=F I x \text { if } 2 / 3<x \leqq 1}
\end{array}\right.
$$

Thus $F$ and I satisfy condition (2.1) and quasi commute. Further, we have that

$$
\begin{aligned}
& \delta(F x, F y)=\max \left\{\frac{x}{x+4}, \frac{y}{y+4}\right\} \leq \frac{1}{2} \cdot \max \left\{\frac{x}{2}, \frac{y}{2}\right\} \\
&=\frac{1}{2} \cdot \max \{\delta(I x, F x), \delta(I y, F y)\}
\end{aligned}
$$

if $x$ and $y$ are in $[0,2 / 3]$ and

$$
\delta(F x, F y)=\max \left\{\frac{x}{x+4}, \frac{y}{y+4}\right\}<\frac{1}{2} \cdot 1=\frac{1}{2} \cdot \max \{\delta(I x, F x), \delta(I y, F y)\}
$$

if at least $x$ or $y$ is in $(2 / 3,1]$. Then the inequality $(2.5)$ is satisfied if one assumes $\psi(t)=t / 2$ for all $t \geqq 0$ and clearly (2.3) holds since $X$ is a bounded metric space. So all the assumptions of Theorem 4 hold but Theorem 2 is not applicable since $F$ and I do not commute. Note that F and I also weakly commute since

$$
\delta(\mathrm{FIx}, \mathrm{IFx})= \begin{cases}\mathrm{x} /(\mathrm{x}+8) \leqq \mathrm{x} / 2=\delta(\mathrm{Ix}, \mathrm{Fx}) & \text { if } 0 \leqq \mathrm{x} \leqq 2 / 3, \\ 1 / 5<1=\delta(\mathrm{Ix}, \mathrm{Fx}) & \text { if } 2 / 3 ; \mathrm{x} \leqq 1,\end{cases}
$$

but Theorem 3 is not applicable since $I$ is not continuous in $X$.

The Example 2 of [2] proves that the weak commutativity is a necessary condition in Theorem 3. Now we prove that the quasi commutativity is a necessary condition in Theorem 4.

EXAMPLE 2. Let $X=\{x, y, z\}$ be a finite set with metric $d$ defined as $d(x, y)=$ $d(x, z)=1, d(y, z)=2$. Define $F$ and $I$ as $F x=F z=\{x\}, F y=\{x, z\}$ and $I x=y, I y=x, I z=z$.

of course $F$ is continuous in $X$ and the conditions (2.1) and (2.3) are trivially

$\delta(F y, F \alpha)=\max \{d(x, y), d(x, z)\}=1=\frac{1}{2} \cdot 2= \begin{cases}\frac{1}{2} \cdot \delta(I x, F y) & \text { if } \alpha=x, \\ \frac{1}{2} \cdot \delta(I z, F y) & \text { if } \alpha=z,\end{cases}$

and $\delta(F x, F z)=0$. Hence the inequality (2.5) is satisfied if $\psi(t)=t / 2$ for all $t \geqq 0$ and then all the assumptions of Theorem 4 hold, except the quasi commutativity since $I F y=I\{y, z\}=\{x, z\} f\{x\}=F x=F I y$ but $F$ and $I$ do not have common fixed points.

The Example 3 of [2] proves that the condition (2.1) is necessary in Theorem 3 . The next example proves that the same condition is necessary in Theorem 4, even if $F$ and I are single-valued mappings.

EXAMPLE 3. Let $X=[0,+\infty)$ with the Euclidean metric $d, F x=x$ for all $x$ in $X$ and $I x=1$ if $x=0, I x=2 x$ if $x>0$. Then we have that $F$ and $I$ (quasi) commute and $F$ is continuous in $X$. The sequence $\left\{d\left(F_{x_{n}}, F_{x_{1}}\right): n=0,1,2, \ldots\right\}$ is bounded for any $x_{0}$ in $X-\{0\}$ since it is easily seen that

$$
I x_{n+1}=F x_{n}=2^{-n} x_{0}
$$

for any $x_{0} \in X-\{0\}$. Further we have that 
$d(F x, F y)= \begin{cases}|x-y|=\frac{1}{2}|2 x-2 y|=\frac{1}{2} d(I x, I y) & \text { if } x>0, y>0, \\ y=\frac{1}{2} \cdot 2 y=\frac{1}{2} \cdot d(I y, F x) & \text { if } x=0, y>0 .\end{cases}$

Thus the inequality (2.5) is satisfied if $\psi(t)=t / 2$ for all $t \geqq 0$ and hence all the assumptions of Theorem 4 hold except the condition (2.1) since $F(X)=X \nsubseteq X-\{0\}=$ $I(X)$, but $I$ does not have fixed points.

The Example 4 of [2] proves that the continuity of $I$ is a necessary condition in Theorem 3. Now we show that the continuity of $F$ is necessary in Theorem 4.

EXAMPLE 4. Let $X=[0,1]$ with the function $\delta$ induced by the Euclidean metric $d$ and define $F$ and $I$ as $F x=1 / 2$ if $x=0$ and $F x=(0, x / 2]$ if $x>0, I x=1$ if $x=0$ and $I x=x$ if $x>0$. Of course, condition (2.3) holds since $X$ is a bounded metric space and (2.1) is satisfied since

$$
F(X)=\left(0, \frac{1}{2}\right] \subseteq(0,1]=I(X) .
$$

Note that $F$ quasi commutes with I since

and

$$
I F 0=I\left(\frac{1}{2}\right)=\frac{1}{2} \in\left(0, \frac{1}{2}\right]=F 1=F I 0
$$

$$
\mathrm{IFx}=\mathrm{I}\left(0, \frac{\mathrm{x}}{2}\right]=\left(0, \frac{\mathrm{x}}{2}\right]=\mathrm{Fx}=\mathrm{FIx}
$$

if $x>0$. Further we have that

$$
\delta(\mathrm{Fx}, \mathrm{Fy})= \begin{cases}\frac{1}{2} \cdot \max \{\mathrm{x}, \mathrm{y}\}=\frac{1}{2} \cdot \max \{\delta(\mathrm{Ix}, \mathrm{Fx}), \delta(\mathrm{Iy}, \mathrm{Fy})\} & \text { if } \mathrm{x}>0, \mathrm{y}>0 \\ \frac{1}{2}=\frac{1}{2} \cdot 1=\frac{1}{2} \cdot \delta(\mathrm{Ix}, \mathrm{Fy}) & \text { if } \mathrm{x}=0, \mathrm{y}>0\end{cases}
$$

Then the inequality (2.5) is satisfied if $\psi(t)=t / 2$ for all $t \geqq 0$ and all the assumptions of Theorem 4 hold except the continuity of F, but F and I do not have common fixed points.

\section{ANOTHER FIXED POINT THEOREM.}

In this Section we establish another result by using a weaker condition than the commutativity between two single-valued mappings of $X$ into itself, but, following the ideas of this work, we give this condition between a set-valued mapping $F$ of $X$ into $B(X)$ and a single-valued mapping $I$ of $X$ into itself. Precisely, we say that $F$ and $I$ slightly commute if $I F x \in B(X)$ and

$$
\delta(F I x, I F x) \leqq \max \{\delta(I x, F x) \text {, diam Fx }\}
$$

for $a 11 \mathrm{x}$ in $\mathrm{X}$. Note that if $\mathrm{F}$ is a single-valued mapping, then diam $\mathrm{Fx}=\mathrm{diam}$ $I F x=0$ for $a 11 x$ in $x$ and hence (2.4) and (4.1) become $d(F I x, I F x) \leqq d(I x, F x)$ for alj $x$ in $X$, that is the condition given in [5].

In the sequel we use the following lemma of [6].

LEMMA 2. Let $\left\{A_{n}\right\}$ be a sequence of nonempty bounded subsets of $(X, d)$ and $y$ be a point of $X$ such that

$$
\lim _{n \rightarrow \infty} \delta\left(A_{n}, y\right)=0
$$

Then the sequence $\left\{A_{n}\right\}$ converges to the set $\{y\}$.

Now we give the following result. 
THEOREM 5. Let $\vec{F}$ be a mapping of $X$ into $B(X)$ and $I$ be a mapping of $X$ into itself satisfying the inequality (2.5) for all $x, y$ in $X$, where $\psi \varepsilon \Psi$. If there exists a point $x_{0}$ in $X$ satisfying condition (2.3), if $F$ and I slightly commute, if (2.1) holds and if $F$ or $I$ is continuous in $X$, then $F$ and $I$ have a unique common $f$ ixed point $z$ and further $F z=\{z\}$.

PROOF. We omit the first part of this proof since it is identical to the first part of the proof of Theorem 3.1 of [2].

As in [2], we can prove that the sequence $\left\{I_{n}\right\}$ converges to a point $z$ in $X$ and the sequence of sets $\left\{F x_{n}\right\}$ converges to the set $\{z\}$. Since $F$ and I slightly commute, we have that

$$
\delta\left(F I x_{n}, I F x_{n}\right) \leqq \max \left\{\delta\left(I x_{n}, F x_{n}\right), \delta\left(F x_{n}, F x_{n}\right)\right\}
$$

for any $n=0,1,2, \ldots$ and as $n \rightarrow \infty$, we deduce by Lemma 1 that

$$
\lim _{n \rightarrow \infty} \delta\left(\operatorname{FIx}_{n}, \operatorname{IFx}_{n}\right)=d(z, z)=0 \text {. }
$$

Now we assume that $F$ is continuous in $X$. Then the sequence of subsets $\left\{F I x_{n}\right\}$ converges to the set $\{F z\}$ and using inequality (2.5), we have that

$$
\begin{aligned}
& \delta\left(F I x_{n+1}, F x_{n}\right) \leqq \psi\left(\operatorname { m a x } \left\{d\left(I^{2} x_{n+1}, I x_{n}\right), \delta\left(I^{2} x_{n+1}, F I x_{n+1}\right), \delta\left(I x_{n}, F x_{n}\right),\right.\right. \\
&\left.\left.\delta\left(I^{2} x_{n+1}, F x_{n}\right), \delta\left(I x_{n}, F I x_{n+1}\right)\right\}\right) \\
& \leqq \psi\left(\operatorname { m a x } \left\{\delta\left(I F x_{n}, I x_{n}\right), \delta\left(I F x_{n}, F I x_{n+1}\right), \delta\left(I x_{n}, F x_{n}\right),\right.\right. \\
&\left.\left.\delta\left(I F x_{n}, F x_{n}\right), \delta\left(I x_{n}, F I x_{n+1}\right)\right\}\right) \\
& \leqq \psi\left(\operatorname { m a x } _ { \{ } \left\{\left(I F x_{n}, F I x_{n}\right)+\delta\left(F I x_{n}, F x_{n}\right)+\delta\left(F x_{n}, I x_{n}\right),\right.\right. \\
&\left.\left.\delta\left(I F x_{n}, F I x_{n}\right)+\delta\left(F I x_{n}, F I x_{n+1}\right), \delta\left(I x_{n}, F x_{n+1}\right)\right\}\right),
\end{aligned}
$$

since $I^{2} x_{n+1}$ is in $\operatorname{IFx}_{n}$ and $\psi$ is nondecreasing.

Since $\psi$ is right continuous, as $n \rightarrow \infty$, using Lemma 1 and (4.2), we obtain that

$$
\delta(F z, z) \leqq \psi(\max \{\delta(F z, z), \delta(F z, F z))\}) .
$$

But again using (2.5) and the nondecreasingness of $\psi$, we deduce that

$$
\begin{aligned}
\delta\left(F_{n+1}, F I x_{n+1}\right) & \leqq \psi\left(\max \left\{d\left(I^{2} x_{n+1}, I^{2} x_{n+1}\right), \delta\left(I^{2} x_{n+1}, F I x_{n+1}\right)\right\}\right) \\
& \leqq \psi\left(\delta\left(\operatorname{IFx}_{n}, F I x_{n}\right)+\delta\left(F I x_{n}, F I x_{n+1}\right)\right)
\end{aligned}
$$

which implies, as $n \rightarrow \infty$, by (4.2) that

$$
\delta(\mathrm{Fz}, \mathrm{Fz}) \leqq \psi(\delta(\mathrm{Fz}, \mathrm{Fz}))
$$

and hence $\delta(F z, F z)=0$ since $\psi(t)<t$ for $t>0$. From (4.3), it follows that $F z=\{z\}$. Since (2.1) holds, there exists a point $w$ in $X$ such that $I w=z$ and using inequality (2.5), we have that

$$
\delta\left(F x_{n}, F w\right) \leqq \psi\left(\max \left\{d\left(I x_{n}, z\right), \delta\left(I x_{n}, F x_{n}\right), \delta(z, F w), \delta\left(I x_{n}, F w\right), \delta\left(z, F x_{n}\right)\right\}\right),
$$

which implies, an $n \rightarrow \infty$, that

$\delta(z, F w) \leqq \psi(\delta H(z, F w))$. 
Thus $F w=z$ and since $F$ and I slightly commute we have that

$\mathrm{d}(\mathrm{Fz}, \mathrm{Iz})=\delta(\mathrm{FIw}, \mathrm{IFw}) \leqq \max \{\delta(\mathrm{Iw}, \mathrm{Fw}), \delta(\mathrm{Fw}, \mathrm{Fw})\}=\mathrm{d}(z, z)=0$.

It follows that $\{z\}=F z=\{I z\}$ and thus $z$ is also a fixed point of $I$.

Now we assume the continuity of $I$ instead of the continuity of $F$. Then the sequence $\left\{I^{2} x_{n}\right\}$ converges to the point $I z$ and the sequence of sets $\left\{I F x_{n}\right\}$ converges to the set $\{\mathrm{Iz}\}$.

We have that

$$
\delta\left(F I x_{n}, I z\right) \leqq \delta\left(F I x_{n}, I F x_{n}\right)+\delta\left(I F x_{n}, I z\right)
$$

and, as $n \rightarrow \infty$, we deduce from $(4.2)$ and Lemma 2 that the sequence of sets $\left\{F_{n} x_{n}\right\}$

also converges to the set $\left\{\mathrm{I}_{2}\right\}$.

Using inequality (2.5) and since $I^{2} x_{n+1}$ is in $I F x_{n}$, we get that

$$
\begin{aligned}
d\left(I^{2} x_{n+1}, I x_{n+1}\right) & \leqq \delta\left(I F x_{n}, F x_{n}\right) \leqq \delta\left(I F x_{n}, F I x_{n}\right)+\delta\left(F I x_{n}, F x_{n}\right) \\
\leqq & \leqq\left(I F x_{n}, F I x_{n}\right)+\psi\left(\operatorname { m a x } \left\{d\left(I^{2} x_{n}, I x_{n}\right), \delta\left(I^{2} x_{n}, F I x_{n}\right),\right.\right. \\
& \left.\left.\delta\left(I x_{n}, F x_{n}\right), \delta\left(I^{2} x_{n}, F x_{n}\right), \delta\left(I x_{n}, F I x_{n}\right)\right\}\right) .
\end{aligned}
$$

As $n \rightarrow \infty$, it follows from (4.2) that

$$
d(I z, z) \leqq \psi(d(I z, z)),
$$

which implies $I z=z$. Using again the inequality $(2.5)$, we have that

$$
\delta\left(F z, F x_{n}\right) \leqq \psi\left(\max \left\{d\left(z, I x_{n}\right), \delta(z, F z), \delta\left(I x_{n}, F x_{n}\right), \delta\left(z, F x_{n}\right), \delta\left(I x_{n}, F z\right)\right\}\right)
$$

and this implies, as $n \rightarrow \infty$, that

$$
\delta(F z, z) \leqq \psi(\delta(F z, z)) .
$$

Then $F z=\{z\}$ and hence $z$ is also a fixed point of $F$. In any case, $z$ is a common fixed point of $F$ and $I$ suppose that $F$ and $I$ have another common fixed point $z '$. Using inequality (2.5), we have that

$$
\mathrm{d}\left(\mathrm{z}, \mathrm{z}^{\prime}\right)=\delta\left(\mathrm{Fz}, \mathrm{Fz} \mathrm{z}^{\prime}\right) \leqq \psi\left(\mathrm{d}\left(\mathrm{z}, \mathrm{z}^{\prime}\right)\right) .
$$

This means that $z=z^{\prime}$ and therefore $z$ is the unique common fixed point of $F$ and I. This completes the proof.

REMARK 1. We note that the mappings $F$ and $I$ of Example 2 satisfy all the assumptions of Theorem 5 except the slight commutativity since $\delta(F I x, I F x)=d(y, z)=2>\max \{1,0\}=\max \{\delta(\operatorname{Ix}, F x), \operatorname{diam} F x\}$.

Therefore the slight commutativity is a necessary condition in Theorem 5 .

REMARK 2. The Example 3 proves that the condition (2.1) is also necessary in Theorem 5 .

REMARK 3. In Example 4, we note that $F$ slightly commutes with I since $\delta(F I O, I F O)=1 / 2=\mathrm{d}(\mathrm{IO}, \mathrm{FO})$ and $\delta(\mathrm{FIx}, \mathrm{IFx})=\mathrm{x} / 2=\mathrm{diam} \mathrm{Fx}$ if $\mathrm{x}>0$. Since all the assumptions of Theorem 5 are satisfied except the continuity of $F$ and $I$ in $X$, we can say that the continuity of $F$ or $I$ in $X$ is a necessary condition in Theorem 5 .

REMARK 4. It is not yet lnown if (2.3) is a necessary condition in Theorems 3,4 and 5 . 
If $F$ is a single-valued mapping, we obtain the resuit of [5] from Theorem 5 .

In Example 1, we explicitly point out that Theorem 5, assuming the continuity of $F$ in $X$, holds good since the mappings $F$ and $I$ are also slightly commuting.

5. CONCLUDING COMMENTS.

Independently from the fixed point considerations until now established, we conclude this paper exhibiting some easy examples which show that the concepts of weak, quasi and slight commutativity between a set-valued mapping and a single-valued mapping do not imply each of the other two.

EXAMPLE 5. Let $X=\{x, y, z\}$ a finite set with function $\delta$ induced by the metric defined as $d(x, y)=d(y, z)=2, d(x, z)=1$. Define $F$ and $I$ as $F x=\{x\}, F y=\{x, z\}, F z=\{y, z\}$ and $I x=x, I y=z, I z=y$. Thus it is easily seen that $F$ and $I$ weakly commute, but they do not quasi commute since $\operatorname{IFy}=\operatorname{I}\{x, z\}=\{x, y\} \subseteq\{y, z\}=F z=F I y$ and further they do not slightly commute since

$$
\delta(F I y, I F y)=2>1=\max \{1,1\}=\max \{\delta(I y, F y), \text { diam Fy } .
$$

EXAMPLE 6. Let $X=\{x, y, z\}$ a finite set with function $\delta$ induced by the metric defined as $d(x, y)=2, d(x, z)=d(y, z)=1$. Let $I$ be as in Example 5 and define $F$ as $F x=\{x\}, F y=F z=\{x, y\}$. Then it is easily seen that $F$ and I slightly commute but they do not quasi commute since $\operatorname{IFy}=\mathrm{I}\{\mathrm{x}, \mathrm{y}\}=\{\mathrm{x}, \mathrm{z}\} \leq\{\mathrm{x}, \mathrm{y}\}=F z=F I y$ and further they do not weakly commute since

$$
\delta(F I y, I F y)=2>1=\max \{1,1\}=\max \{\delta(I y, F y), \operatorname{diam} I F y\} .
$$

EXAMPLE 7. Let $(X, d)$ be as in Example 6 and define $F$ and $I$ as $F x=\{x\}$, $F y=\{x, y\}, F z=\{y\}$ and $I x=x, I y=I z=y$. Thus $F$ and $I$ quasi commute but they do not slightly nor weakly commute since

$$
\delta(\mathrm{FI} z, \mathrm{IFz})=2>0=\max \{\delta(\mathrm{I} z, \mathrm{Fz}), \operatorname{diam} \mathrm{Fz}, \mathrm{diam} I F z\} .
$$

\section{REFERENCES}

1. FISHER, B. Common Fixed Point Theorems for Mappings and Set-Valued Mappings, Rostock. Math. Kolloq. 18 (1981), 69-77.

2. SESSA, S., KHAN, M.S. and IMDAD, M. Common Fixed Point Theorem with a Weak Commutativity Condition, Glasnik Mat. 21(41) (1986), 225-235.

3. FISHER, B. Fixed Points of Mappings and Set-Valued Mappings, J. Univ. Kuwait Sci. $9(1982), 175-180$.

4. ITOH, S. and TAKAHASHI, W. Single-Valued Mappings, Multi-Valued Mappings and Fixed Point Theorems, J. Math. Anal. Appl. 59(1977), 514-521.

5. SESSA, S. On a Weak Commutativity Condition of Mappings in Fixed Point Considerations, Pub1. Inst. Math. 32 (46) (1982), 149-153.

6. SESSA, S. and FISHER, B. Common Fixed Point Theorems for Weakly Commuting Mappings, submitted. 


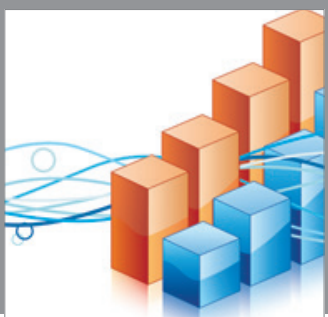

Advances in

Operations Research

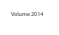

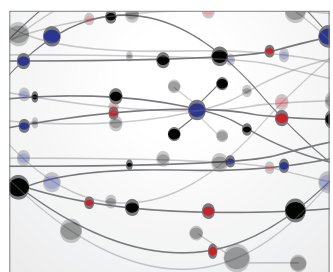

\section{The Scientific} World Journal
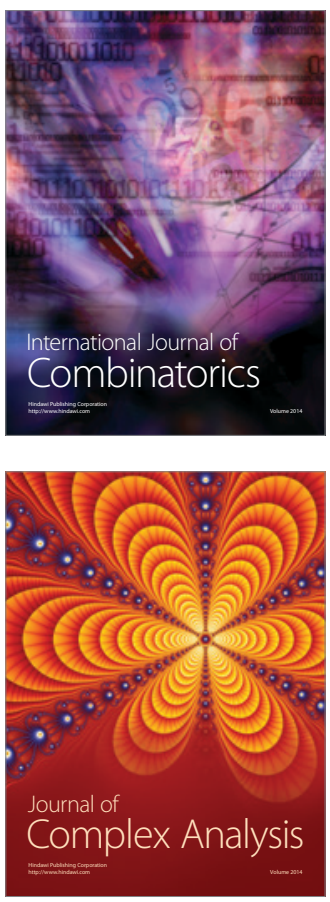

International Journal of

Mathematics and

Mathematical

Sciences
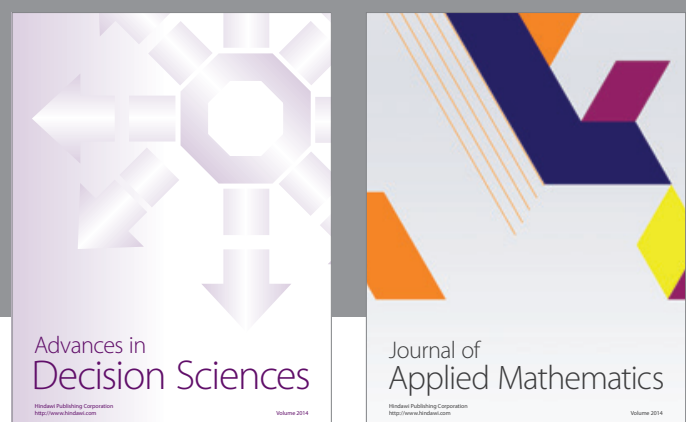

Journal of

Applied Mathematics
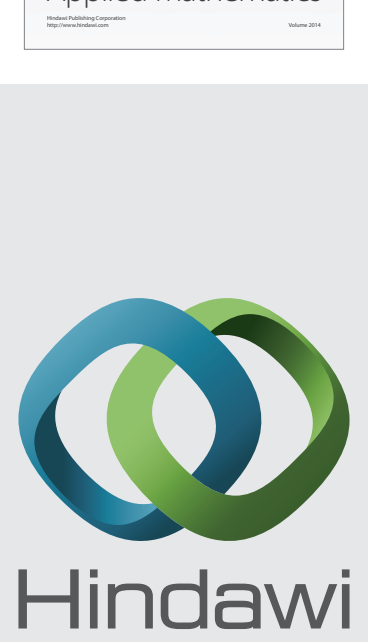

Submit your manuscripts at http://www.hindawi.com
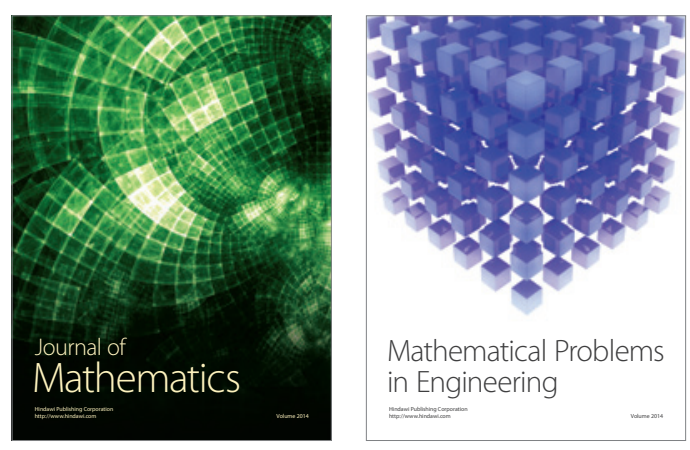

Mathematical Problems in Engineering
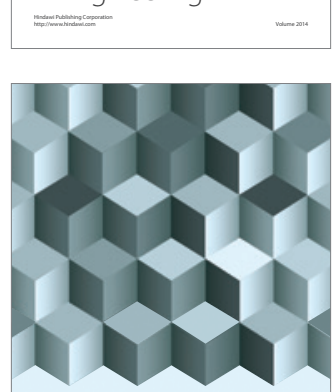

Journal of

Function Spaces
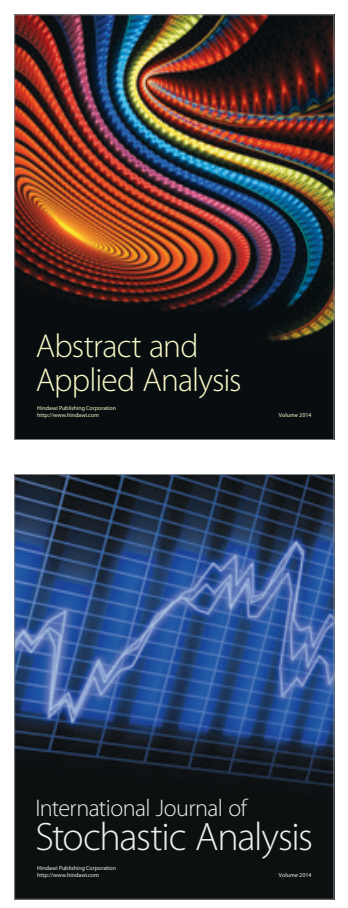

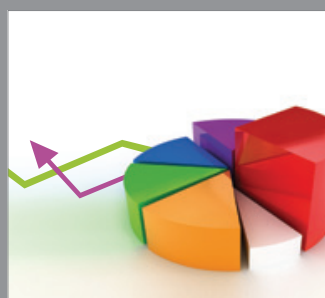

ournal of

Probability and Statistics

Promensencen
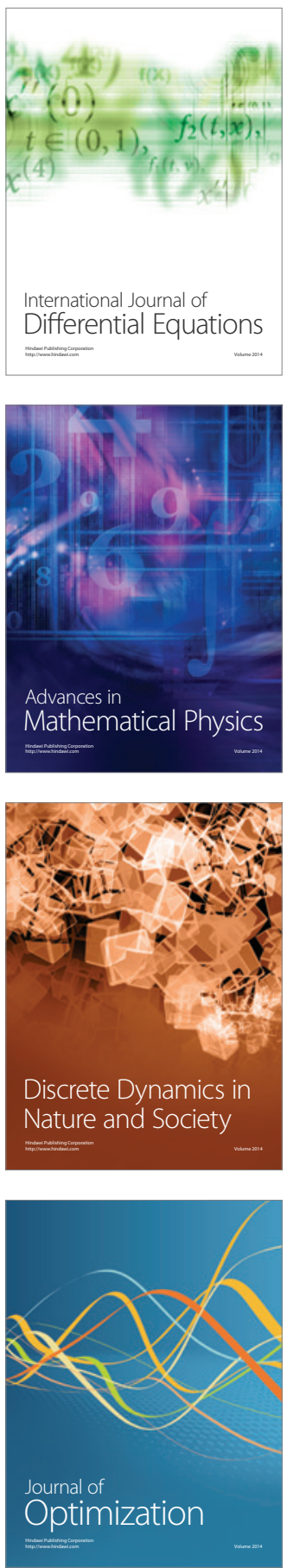